\title{
Mixed Convection Boundary Layer Flow from a Solid Sphere with Newtonian Heating in a Micropolar Fluid
}

\author{
M. Z. Salleh, ${ }^{1}$ R. Nazar, ${ }^{2}$ and I. Pop $^{3}$ \\ ${ }^{1}$ Faculty of Industrial Science and Technology, Universiti Malaysia Pahang, Lebuhraya Tun Razak, \\ 26300 UMP Kuantan, Pahang, Malaysia \\ ${ }^{2}$ School of Mathematical Sciences, Faculty of Science and Technology, Universiti Kebangsaan Malaysia, \\ 43600 UKM Bangi, Selangor, Malaysia \\ ${ }^{3}$ Faculty of Mathematics, University of Cluj, CP 2533400 Cluj, Romania
}

Correspondence should be addressed to R. Nazar, rmn72my@yahoo.com

Received 27 September 2009; Revised 2 December 2009; Accepted 28 December 2009

Copyright (๑) 2010 M. Z. Salleh et al. This is an open access article distributed under the Creative Commons Attribution License, which permits unrestricted use, distribution, and reproduction in any medium, provided the original work is properly cited.

The steady mixed convection boundary layer flow from a solid sphere in a micropolar fluid, generated by Newtonian heating in which the heat transfer from the surface is proportional to the local surface temperature, is considered. The governing boundary layer equations are first transformed into a system of nondimensional equations via the non-dimensional variables, and then into nonsimilar equations before they are solved numerically using an implicit finite-difference scheme known as the Keller-box method. Numerical solutions are obtained for the skin friction coefficient, wall temperature and heat transfer coefficient, as well as the velocity and temperature profiles with several parameters considered, namely the mixed convection parameter, the material or micropolar parameter, and the Prandtl number.

\section{Introduction}

The essence of the theory of micropolar fluid flow lies in the extension of the constitutive equation for Newtonian fluid, so that more complex fluids such as particle suspensions, liquid crystal, animal blood, lubrication, and turbulent shear flows can be described by this theory. The theory of micropolar fluid was first proposed by Eringen [1]. Extensive review of the theory and applications can be found in the review article by Ariman et al. [2] and quite recent papers by Rees and Bassom [3], Pop et al. [4], Nazar et al. [5-8], and so forth. This clearly shows the fast development of the theory of micropolar fluid. In summary, all the papers above considered the boundary condition of either a constant wall temperature or constant heat flux.

Generally, in modeling the boundary layer flow and heat transfer of these problems, the boundary conditions that are usually applied are either a constant wall temperature (CWT) or a constant wall heat flux (CHF). However, there is a class of boundary layer flow and heat transfer problems in which the surface heat transfer depends on the surface temperature. Perhaps the simplest case of which is when there is a linear relation between the surface heat transfer and surface temperature. This situation arises in conjugate heat transfer problems (see, e.g., Merkin and Pop [9]), and also when there is Newtonian heating of the convective fluid from the surface. The latter case has been first discussed in detail by Merkin [10]. The situation with Newtonian heating arises in what are usually termed conjugate convective flows, where the heat is supplied to the convecting fluid through a bounding surface with a finite heat capacity. This results in the heat transfer rate through the surface being proportional to the local difference in the temperature with the ambient conditions. However, the Newtonian heating conditions have been used only recently by Lesnic et al. [11-13] and Pop et al. [14] to study the free convection boundary layer over vertical and horizontal surfaces as well as over a small inclined flat plate from the horizontal surface embedded in a porous medium. The asymptotic solution near the leading edge and the full numerical solution along the whole plate domain have been obtained numerically, whilst the asymptotic solution far downstream along the plate has been obtained analytically. Chaudhary and Jain $[15,16]$ studied the unsteady free convection boundary layer flow past an 


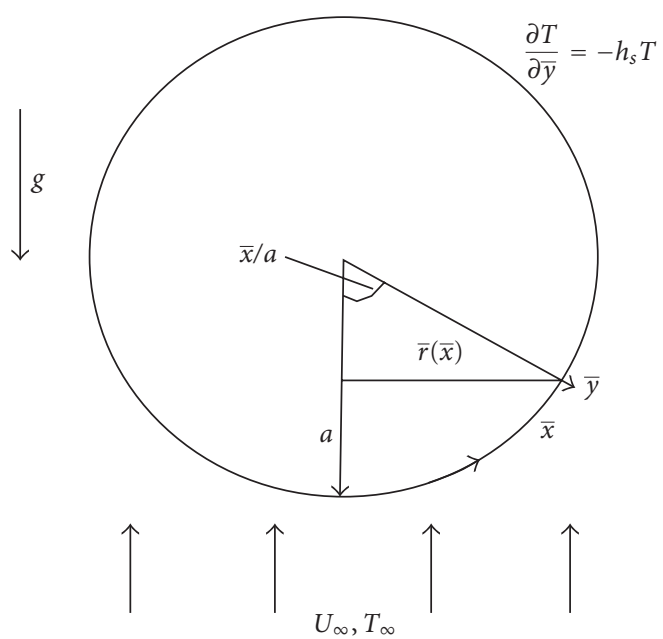

Figure 1: Physical model and coordinate system.

impulsively started vertical infinite flat plate with Newtonian heating.

This configuration occurs in many important engineering devices, for example, in heat exchanger where the conduction in solid tube wall is greatly influenced by the convection in the fluid flowing over it. Furthermore, for conjugate heat transfer around fins where the conduction within the fin and the convection in the fluid surrounding it must be simultaneously analyzed in order to obtain the vital design information and also in convection flow setup when the bounding surfaces absorb heat by solar radiation $[15,16]$. This results in the heat transfer rate through the surface being proportional to the local difference in the temperature with the ambient conditions.

Recently, Salleh et al. [17, 18] employed an implicit finite-difference scheme, namely the Keller-box method to obtain numerical solutions for mixed convection boundary layer flow near the lower stagnation point of a solid sphere and forced convection boundary layer flow at a forward stagnation point with Newtonian heating in viscous fluids, respectively.

Therefore, the aim of the present paper is to study the problem of mixed convection boundary layer flow from a solid sphere with Newtonian heating in a micropolar fluid. The governing boundary layer equations are first transformed into a system of non-dimensional equations via the non-dimensional variables, and then into non-similar equations before they are solved numerically by the Kellerbox method, as described in the books by $\mathrm{Na}$ [19] and Cebeci and Bradshaw [20]. To the best of our knowledge, this present problem (for the case of Newtonian heating) has not been considered before, so that the reported results are new.

\section{Analysis}

Consider a heated sphere of radius $a$, which is immersed in a viscous and incompressible micropolar fluid of ambient temperature $T_{\infty}$, which is subjected to a Newtonian heating $(\mathrm{NH})$ as shown in Figure 1. The convective forced flow is assumed to be moving upward, while the gravity vector $\mathrm{g}$ acts downward in the opposite direction, where the coordinates $\bar{x}$ and $\bar{y}$ are chosen such that $\bar{x}$ measures the distance along the surface of the sphere from the lower stagnation point and $\bar{y}$ measures the distance normal to the surface of the sphere. We assume that the equations are subjected to a Newtonian heating of the form proposed by Merkin [10]. Under the Boussinesq and boundary layer approximations, the basic equations are (Nazar et al. $[6,8])$

$$
\begin{gathered}
\frac{\partial}{\partial \bar{x}}(\bar{r} \bar{u})+\frac{\partial}{\partial \bar{y}}(\bar{r} \bar{v})=0 \\
\bar{u} \frac{\partial \bar{u}}{\partial \bar{x}}+\bar{v} \frac{\partial \bar{u}}{\partial \bar{y}}=\bar{u}_{e} \frac{d \bar{u}_{e}}{d \bar{x}}+\left(\frac{\mu+\kappa}{\rho}\right) \frac{\partial^{2} \bar{u}}{\partial \bar{y}^{2}} \pm g \beta\left(T-T_{\infty}\right) \sin \left(\frac{\bar{x}}{a}\right) \\
+\frac{\kappa}{\rho} \frac{\partial \bar{H}}{\partial \bar{y}}, \\
\rho j\left(\bar{u} \frac{\partial \bar{H}}{\partial \bar{x}}+\bar{v} \frac{\partial \bar{H}}{\partial \bar{y}}\right)=-\kappa\left(2 \bar{H}+\frac{\partial \bar{u}}{\partial \bar{y}}\right)+\gamma \frac{\partial^{2} \bar{H}}{\partial \bar{y}^{2}}, \\
\bar{u} \frac{\partial T}{\partial \bar{x}}+\bar{v} \frac{\partial T}{\partial \bar{y}}=\frac{\nu}{\operatorname{Pr}} \frac{\partial^{2} T}{\partial \bar{y}^{2}}
\end{gathered}
$$

subject to the boundary conditions

$$
\begin{aligned}
& \bar{u}=\bar{v}=0, \quad \frac{\partial T}{\partial \bar{y}}=-h_{s} T(\mathrm{NH}) \quad \text { or } \quad T=T_{w}(\mathrm{CWT}) \\
& \text { or } \quad \frac{\partial T}{\partial \bar{y}}=-\frac{q_{w}}{k}(\mathrm{CHF}), \quad \bar{H}=-n \frac{\partial \bar{u}}{\partial \bar{y}} \quad \text { on } \bar{y}=0 \\
& \bar{u} \longrightarrow \bar{u}_{e}(\bar{x}), \quad T \longrightarrow T_{\infty}, \quad \bar{H} \longrightarrow 0 \quad \text { as } \bar{y} \longrightarrow \infty,
\end{aligned}
$$

where $\bar{u}$ and $\bar{v}$ are the velocity components along the $\bar{x}$ and $\bar{y}$ directions, respectively, $\bar{H}$ is the microrotation component normal to the $\bar{x}-\bar{y}$ plane, $\gamma$ is the spin gradient viscosity, $\kappa$ is the vortex viscosity, $T$ is the local temperature, $T_{w}$ is the wall temperature, $g$ is the gravity acceleration, $\alpha=$ $v / \mathrm{Pr}$ is the thermal diffusivity, $\beta$ is the thermal expansion coefficient, $v=\mu / \rho$ is the kinematic viscosity, $\mu$ is the dynamic viscosity, $\rho$ is the density, $\operatorname{Pr}$ is the Prandtl number, $k$ is the thermal conductivity, $q_{w}$ is the constant heat flux from the wall, and $h_{s}$ is the heat transfer parameter. It is worth mentioning that in boundary conditions (2), $n$ is a constant and $0 \leq n \leq 1$. The value $n=0$, which indicates $\bar{H}=0$ at the wall, represents concentrated particle flows in which the particle density is sufficiently great that microelements close to the wall are unable to rotate or called as "strong" concentration of microelements [21, 22]. The case corresponding to $n=1 / 2$ results in the vanishing of antisymmetric part of the stress tensor and represents "weak" concentration of microelements [23]. In this case, the particle rotation is equal to fluid vorticity at the boundary for fine particle suspension. When $n=1$, we have flows which are representative of turbulent boundary layer [24]. The case of $n=1 / 2$ is considered in the present study. 
Let $\bar{r}(\bar{x})$ be the radial distance from the symmetrical axis to the surface of the sphere and $\bar{u}_{e}$ the local free stream velocity, which are given by

$$
\begin{gathered}
\bar{r}(\bar{x})=a \sin \left(\frac{\bar{x}}{a}\right), \quad \bar{u}_{e}(\bar{x})=\frac{3}{2} U_{\infty} \sin \left(\frac{\bar{x}}{a}\right), \\
\gamma=\left(\mu+\frac{\kappa}{2}\right) j,
\end{gathered}
$$

where $j$ is the micro-inertia per unit mass.

We introduce now the following non-dimensional variables:

$x=\frac{\bar{x}}{a}, \quad y=\operatorname{Re}^{1 / 2}\left(\frac{\bar{y}}{a}\right), \quad u=\frac{\bar{u}}{U_{\infty}}$,

$v=\operatorname{Re}^{1 / 2}\left(\frac{\bar{v}}{U_{\infty}}\right), \quad H=\left(\frac{a}{U_{\infty}}\right) \operatorname{Re}^{-1 / 2} \bar{H}, \quad u_{e}(x)=\frac{\bar{u}_{e}(\bar{x})}{U_{\infty}}$,

$h_{s}=\frac{\mathrm{Re}^{1 / 2}}{a}, \quad r(x)=\frac{\bar{r}(\bar{x})}{a}, \quad \theta=\frac{T-T_{\infty}}{T_{\infty}}(\mathrm{NH}) \quad$ or

$\theta=\frac{T-T_{\infty}}{T_{w}-T_{\infty}}(\mathrm{CWT}) \quad$ or $\quad \theta=\left(\frac{k}{a q_{w}}\right) \operatorname{Re}^{1 / 2}\left(T-T_{\infty}\right)(\mathrm{CHF})$,

where $\operatorname{Re}=U_{\infty} a / v$ is the Reynolds number. Using (4), the system of (1) then becomes

$$
\begin{gathered}
\frac{\partial}{\partial x}(r u)+\frac{\partial}{\partial y}(r v)=0 \\
u \frac{\partial u}{\partial x}+v \frac{\partial u}{\partial y}=u_{e} \frac{d u_{e}}{d x}+(1+K) \frac{\partial^{2} u}{\partial y^{2}}+K \frac{\partial H}{\partial y}+\lambda \theta \sin x \\
u \frac{\partial H}{\partial x}+v \frac{\partial H}{\partial y}=-K\left(2 H+\frac{\partial u}{\partial y}\right)+\left(1+\frac{K}{2}\right) \frac{\partial^{2} H}{\partial y^{2}} \\
u \frac{\partial \theta}{\partial x}+v \frac{\partial \theta}{\partial y}=\frac{1}{\operatorname{Pr}} \frac{\partial^{2} \theta}{\partial y^{2}}
\end{gathered}
$$

subject to the boundary conditions

$$
\begin{gathered}
u=v=0, \quad \frac{\partial \theta}{\partial y}=-(1+\theta)(\mathrm{NH}), \quad \text { or } \quad \theta=1(\mathrm{CWT}) \\
\text { or } \quad \frac{\partial \theta}{\partial y}=-1(\mathrm{CHF}), \quad H=-\frac{1}{2} \frac{\partial u}{\partial y} \quad \text { on } y=0, \\
u \longrightarrow u_{e}(x), \quad \theta \longrightarrow 0, \quad H \longrightarrow 0 \quad \text { as } y \longrightarrow \infty,
\end{gathered}
$$

where $\lambda$ is the mixed convection parameter and $K$ is the material parameter which are given by

$$
\begin{gathered}
\lambda=\frac{G r}{\mathrm{Re}^{2}}(\mathrm{NH} \text { and } \mathrm{CWT}) \quad \text { or } \lambda=\frac{G r}{\mathrm{Re}^{5 / 2}}(\mathrm{CHF}), \\
K=\frac{\kappa}{\mu}
\end{gathered}
$$

with $G r=g \beta T_{\infty} a^{3} / \nu^{2}(\mathrm{NH})$ or $G r=g \beta\left(T_{w}-T_{\infty}\right) a^{3} / v^{2}$ $(\mathrm{CWT})$ or $G r=g \beta\left(a q_{w} / k\right) a^{3} / v^{2}$ (CHF) being the Grashof number. It is worth mentioning that in both cases of CWT and CHF, $\lambda>0$ is for the aiding or assisting flow (heated sphere) and $\lambda<0$ is for the opposing flow (cooled sphere), while for the present case of $\mathrm{NH}$, the value of $\lambda$ considered is only for $\lambda>0$.

Further, the stream function $\psi$ is introduced and it is defined as

$$
u=\frac{1}{r} \frac{\partial \psi}{\partial y}, \quad v=-\frac{1}{r} \frac{\partial \psi}{\partial x} .
$$

Thus, since $\sin x / x \rightarrow 1$ as $x \rightarrow 0$, it is appropriate to introduce the transformation

$$
\psi=x r(x) f(x, y), \quad \theta=\theta(x, y), \quad H=x h(x, y) .
$$

Equations (6) to (8) then become

$$
\begin{aligned}
& (1+K) \frac{\partial^{3} f}{\partial y^{3}}+\left(1+\frac{x}{\sin x} \cos x\right) f \frac{\partial^{2} f}{\partial y^{2}}-\left(\frac{\partial f}{\partial y}\right)^{2}+\frac{9}{4} \frac{\sin x \cos x}{x} \\
& +K \frac{\partial h}{\partial y}+\lambda \frac{\sin x}{x} \theta=x\left(\frac{\partial f}{\partial y} \frac{\partial^{2} f}{\partial x \partial y}-\frac{\partial f}{\partial x} \frac{\partial^{2} f}{\partial y^{2}}\right), \\
& \left(1+\frac{K}{2}\right) \frac{\partial^{2} h}{\partial y^{2}}+\left(1+\frac{x}{\sin x} \cos x\right) f \frac{\partial f}{\partial y}-\frac{\partial f}{\partial y} h-K\left(2 h+\frac{\partial^{2} f}{\partial y^{2}}\right) \\
& =x\left(\frac{\partial f}{\partial y} \frac{\partial h}{\partial x}-\frac{\partial f}{\partial x} \frac{\partial h}{\partial y}\right), \\
& \frac{1}{\operatorname{Pr}} \frac{\partial^{2} \theta}{\partial y^{2}}+\left(1+\frac{x}{\sin x} \cos x\right) f \frac{\partial \theta}{\partial y}=x\left(\frac{\partial f}{\partial y} \frac{\partial \theta}{\partial x}-\frac{\partial f}{\partial x} \frac{\partial \theta}{\partial y}\right)
\end{aligned}
$$

subject to the boundary conditions

$$
\begin{gathered}
f=\frac{\partial f}{\partial y}=0, \quad \frac{\partial \theta}{\partial y}=-(1+\theta)(\mathrm{NH}) \quad \text { or } \quad \theta=1(\mathrm{CWT}) \\
\text { or } \quad \frac{\partial \theta}{\partial y}=-1(\mathrm{CHF}), \quad h=-\frac{1}{2} \frac{\partial^{2} f}{\partial y^{2}} \quad \text { on } y=0 \\
\frac{\partial f}{\partial y} \longrightarrow \frac{3}{2} \frac{\sin x}{x}, \quad \theta \longrightarrow 0, \quad h \longrightarrow 0 \quad \text { as } y \longrightarrow \infty .
\end{gathered}
$$

At the lower stagnation point of the sphere, $x \approx 0,(13)$ reduces to the following ordinary differential equations:

$$
\begin{aligned}
& (1+K) f^{\prime \prime \prime}+2 f f^{\prime \prime}-f^{\prime 2}+K h^{\prime}+\lambda \theta+\frac{9}{4}=0 \\
& \left(1+\frac{K}{2}\right) h^{\prime \prime}+2 f h^{\prime}-f^{\prime} h-K\left(2 h+f^{\prime \prime}\right)=0
\end{aligned}
$$

$$
\frac{1}{\operatorname{Pr}} \theta^{\prime \prime}+2 f \theta^{\prime}=0
$$


and the boundary conditions (14) become

$$
\begin{gathered}
f(0)=f^{\prime}(0)=0, \quad \theta^{\prime}(0)=-(1+\theta(0))(\mathrm{NH}) \text { or } \\
\theta(0)=1(\mathrm{CWT}) \quad \text { or } \quad \theta^{\prime}(0)=-1(\mathrm{CHF}), \\
h=-\frac{1}{2} f^{\prime \prime}(0) \quad \text { on } y=0, \\
f^{\prime} \longrightarrow \frac{3}{2}, \quad \theta \longrightarrow 0, h \longrightarrow 0 \quad \text { as } y \longrightarrow \infty,
\end{gathered}
$$

where primes denote differentiation with respect to $y$.

The quantities of physical interest are the skin friction coefficient, $C_{f}$ and the local wall temperature distribution, $\theta_{w}(x)$, which are given by

$$
\begin{aligned}
& \operatorname{Re}^{1 / 2} C_{f}=x\left(1+\frac{K}{2}\right)\left(\frac{\partial^{2} f}{\partial y^{2}}\right)_{y=0}, \\
& \theta_{w}(x)=-1-\left(\frac{\partial \theta}{\partial y}\right)_{y=0}(\mathrm{NH}),
\end{aligned}
$$

where $C_{f}=\tau_{w} / \rho U_{\infty}^{2}$ and $\tau_{w}=\mu(\partial \bar{u} / \partial \bar{y})_{\bar{y}=0}$ is the wall skin friction or wall shear stress.

\section{Solution Procedure}

Equations in (13) subject to the boundary conditions (14) are solved numerically using the Keller-box method as described in the books by $\mathrm{Na}$ [19] and Cebeci and Bradshaw [20]. The solution is obtained in the following four steps.

(i) Reduce (13) to a first-order system.

(ii) Write the difference equations using central differences.

(iii) Linearize the resulting algebraic equations by Newton's method, and write them in the matrix-vector form.

(iv) Solve the linear system by the block tridiagonal elimination technique (see Salleh et al. [25] for the details of this method).

\section{Results and Discussion}

Equations in (13) subject to the boundary conditions (14) are solved numerically using an efficient implicit finitedifference scheme with various parameters, namely the mixed convection or buoyancy parameter $\lambda$, the material or micropolar parameter $K$ and the Prandtl number Pr. In this paper, the numerical solutions start at the lower stagnation point of the sphere, $x \approx 0$, as given in (15) subjects to the boundary conditions (16) and proceed round the sphere up to the separation point $x_{s}$. The values of the skin friction coefficient, $C_{f}$ and the local wall temperature distribution, $\theta_{w}(x)$ have been obtained at different positions $0 \leq x \leq$ $120^{\circ}$ for following values of the material parameter $K=$ 0 (Newtonian fluid), 1, 2, and 3 (micropolar fluid), with
TABle 1: Values of $f^{\prime \prime}(0)$ and $-\theta^{\prime}(0)$ for various values of $\lambda$ with $\operatorname{Pr}=7$ and $K=1$ (CWT).

\begin{tabular}{lcccc}
\hline$\lambda$ & \multicolumn{2}{c}{$f^{\prime \prime}(0)$} & \multicolumn{2}{c}{$-\theta^{\prime}(0)$} \\
& Nazar et al. [8] & Present & Nazar et al. [8] & Present \\
\hline-5.0 & 1.0549 & 1.0462 & 1.5834 & 1.5681 \\
-2.0 & 1.4465 & 1.4430 & 1.6726 & 1.6581 \\
0.0 & 1.7042 & 1.7020 & 1.7275 & 1.7127 \\
3.0 & 2.1018 & 2.1089 & 1.8081 & 1.7944 \\
10.0 & 2.9568 & 2.9554 & 1.9617 & 1.9434 \\
\hline
\end{tabular}

TABLe 2: Values of $f^{\prime \prime}(0)$ and $\theta(0)$ for various values of $\lambda$ with $\operatorname{Pr}=$ 0.7 and $K=1$ (CHF).

\begin{tabular}{lcccc}
\hline$\lambda$ & $f^{\prime \prime}(0)$ & \multicolumn{2}{c}{$\theta(0)$} \\
& Nazar et al. [6] & Present & Nazar et al. [6] & Present \\
\hline-3.0 & 0.3383 & 0.3035 & 1.6592 & 1.6781 \\
-2.0 & 0.9790 & 0.9725 & 1.4674 & 1.4708 \\
0.0 & 1.7135 & 1.7112 & 1.3255 & 1.3274 \\
2.0 & 2.2941 & 2.2923 & 1.2455 & 1.2471 \\
10.0 & 4.0030 & 3.9911 & 1.0889 & 1.0909 \\
\hline
\end{tabular}

TABle 3: Values of $f^{\prime \prime}(0)$ and $\theta(0)$ for various values of $\lambda$ with fixed $\mathrm{Pr}=1$ and $K=0$ (viscous fluid) $(\mathrm{NH})$.

\begin{tabular}{lcccc}
\hline$\lambda$ & \multicolumn{2}{c}{$f^{\prime \prime}(0)$} & \multicolumn{2}{r}{$\theta(0)$} \\
& Salleh et al. [17] & Present & Salleh et al. [17] & Present \\
\hline 0.05 & 3.5485 & 3.5485 & 65.4268 & 65.4268 \\
1.0 & 5.2161 & 5.2162 & 8.7364 & 8.7367 \\
5.0 & 8.1273 & 8.1273 & 3.9495 & 3.9495 \\
7.0 & 9.1151 & 9.1151 & 3.4047 & 3.4047 \\
10.0 & 10.3730 & 10.3730 & 2.9258 & 2.9258 \\
\hline
\end{tabular}

various values of the mixed convection parameter $\lambda$. It should be noticed that the present results were obtained up to the value of $x=120^{\circ}$, as given by Nazar et al. [6,8]. Values of $\operatorname{Pr}$ considered are $\operatorname{Pr}=0.7,1$, and 7 which correspond to air, electrolyte solution and water, respectively.

Due to the decoupled boundary layer equation (15) when the mixed convection parameter $\lambda=0$ and $K=0$, there is a unique value of the reduced skin friction coefficient, $f^{\prime \prime}(0)=2.4104$ for all Prandtl number Pr, which is in good agreement with the value $f^{\prime \prime}(0)=2.4151$ found by Nazar et al. [6-8]. The values of $f^{\prime \prime}(0),-\theta^{\prime}(0)$ and $\theta(0)$ for the cases of CWT, CHF, and NH are shown in Tables 1, 2, and 3 , respectively. Some results reported by Nazar et al. $[6,8]$ for the cases of CWT and CHF and Salleh et al. [17] for the case of $\mathrm{NH}$ in viscous fluid $(K=0)$ are also included in these tables. It is found that the agreement between the previously published results with the present ones is very good. We can conclude that this method works efficiently and we are also confident that the results presented here are accurate. 
TABLE 4

(a) Comparison on the values of $\theta(0)$ and $-\theta^{\prime}(0)$ from the present solution of (15) for different boundary conditions with $\operatorname{Pr}=7$ and $K=1$ (micropolar fluid).

\begin{tabular}{lcccc}
\hline$\lambda$ & CWT & CHF & \multicolumn{2}{c}{ NH } \\
& $-\theta^{\prime}(0)$ & $\theta(0)$ & $\theta(0)$ & $-\theta^{\prime}(0)$ \\
\hline-2.0 & 1.6670 & 0.5878 & - & - \\
-1.0 & 1.6980 & 0.5847 & - & - \\
0.0 & 1.7272 & 0.5790 & 1.2748 & 2.2748 \\
2.0 & 1.7805 & 0.5688 & 1.2410 & 2.2410 \\
4.0 & 1.8286 & 0.5598 & 1.1750 & 2.1750 \\
6.0 & 1.8725 & 0.5517 & 1.1190 & 2.1190 \\
8.0 & 1.9159 & 0.5445 & 1.0745 & 2.0745 \\
10.0 & 1.9509 & 0.5379 & 1.0378 & 2.0378 \\
20.0 & 2.1101 & 0.5115 & 0.9165 & 1.9165 \\
\hline
\end{tabular}

(b) Comparison on the values of $f^{\prime \prime}(0)$ from the present solution of (15) for different boundary conditions with $\operatorname{Pr}=7$ and $K=1$ (micropolar fluid).

\begin{tabular}{lllc}
\hline$\lambda$ & CWT & CHF & NH \\
& $f^{\prime \prime}(0)$ & $f^{\prime \prime}(0)$ & $f^{\prime \prime}(0)$ \\
\hline-2.0 & 1.4748 & 1.6244 & - \\
-1.0 & 1.6179 & 1.6761 & - \\
0.0 & 1.7568 & 1.7568 & 1.7568 \\
2.0 & 2.0236 & 1.9102 & 2.1176 \\
4.0 & 2.2785 & 2.0547 & 2.3937 \\
6.0 & 2.5235 & 2.1918 & 2.6367 \\
8.0 & 2.7603 & 2.3227 & 2.8568 \\
10.0 & 2.9901 & 2.4483 & 3.0597 \\
20.0 & 4.0590 & 3.0160 & 3.9136 \\
\hline
\end{tabular}

Tables 4(a) and 4(b) show comparisons on the values of $\theta(0)$ and $-\theta^{\prime}(0)$ as well as $f^{\prime \prime}(0)$, respectively, for different boundary conditions (CWT, CHF, and NH cases) with fixed $\operatorname{Pr}=7$ and $K=1$ (micropolar fluid). Table 4(a) shows the values of the heat transfer coefficient $-\theta^{\prime}(0)$ for the case of CWT, the wall temperature $\theta(0)$ for the case of CHF, and the wall temperature $\theta(0)$ or the heat transfer coefficient $-\theta^{\prime}(0)$ for the case of NH. It is noticed from Table 4(a) that for the case of CWT, as $\lambda$ increases, the heat transfer coefficient $-\theta^{\prime}(0)$ also increases. This table also shows that for the case of CHF, as $\lambda$ increases, the wall temperature $\theta(0)$ decreases. However, for the case of $\mathrm{NH}$, as $\lambda$ increases, both the heat transfer coefficient and the wall temperature decrease. The trend for NH case is similar to the CHF case but different from the CWT case. On the other hand, Table 4(b) shows the values of the skin friction coefficient $f^{\prime \prime}(0)$ for the cases of CWT, CHF, and NH. It shows that for all the three cases, as $\lambda$ increases, the skin friction coefficient $f^{\prime \prime}(0)$ also increases. The trend for NH case is similar to the CWT and CHF cases, but the values of $f^{\prime \prime}(0)$ are closer to the CWT case than the CHF case.
TABle 5: Values of $f^{\prime \prime}(0)$ and $\theta(0)$ for various values of $\lambda$ and $K$ with fixed $\operatorname{Pr}=7(\mathrm{NH})$.

\begin{tabular}{lcccccc}
\hline \multirow{2}{*}{$\lambda$} & \multicolumn{2}{c}{$K=1$} & \multicolumn{2}{c}{$K=2$} & \multicolumn{2}{c}{$K=3$} \\
& $f^{\prime \prime}(0)$ & $\theta(0)$ & $f^{\prime \prime}(0)$ & $\theta(0)$ & $f^{\prime \prime}(0)$ & $\theta(0)$ \\
\hline 0.05 & 1.7662 & 1.3736 & 1.4597 & 1.5677 & 1.2777 & 1.7382 \\
1.0 & 1.9332 & 1.2988 & 1.5980 & 1.4970 & 1.3990 & 1.6551 \\
5.0 & 2.4950 & 1.1527 & 2.0583 & 1.3055 & 1.7992 & 1.4333 \\
7.0 & 2.7268 & 1.1019 & 2.2469 & 1.2450 & 1.9623 & 1.3643 \\
10.0 & 3.0386 & 1.0378 & 2.5001 & 1.1755 & 2.1807 & 1.2853 \\
\hline
\end{tabular}

TABLe 6: Values of $f^{\prime \prime}(0)$ and $\theta(0)$ for various values of $\lambda$ and $\operatorname{Pr}$ with fixed $K=2(\mathrm{NH})$.

\begin{tabular}{lcccccc}
\hline \multirow{2}{*}{$\lambda$} & \multicolumn{2}{c}{$\operatorname{Pr}=0.7$} & \multicolumn{2}{c}{$\operatorname{Pr}=1$} & \multicolumn{2}{c}{$\operatorname{Pr}=7$} \\
& $f^{\prime \prime}(0)$ & $\theta(0)$ & $f^{\prime \prime}(0)$ & $\theta(0)$ & $f^{\prime \prime}(0)$ & $\theta(0)$ \\
\hline 0.05 & 5.3893 & 582.9966 & 3.3508 & 269.9892 & 1.4597 & 1.5677 \\
1.0 & 5.9345 & 33.9767 & 4.0100 & 18.9700 & 1.5980 & 1.4970 \\
5.0 & 7.5188 & 9.7701 & 5.5475 & 6.5872 & 2.0583 & 1.3055 \\
7.0 & 8.1221 & 7.8312 & 6.0922 & 5.4627 & 2.2469 & 1.2450 \\
10.0 & 8.9099 & 6.2842 & 6.7908 & 4.5284 & 2.5001 & 1.1755 \\
\hline
\end{tabular}

The values of $f^{\prime \prime}(0)$ and $\theta(0)$ for various values of $\lambda$, $K$ and $P r$ are shown in Tables 5 and 6, respectively. From Table 5 , it is found that as $K$ increases, the values of $f^{\prime \prime}(0)$ are higher and $\theta(0)$ are lower for $K=1$ than those for $K=2$ and 3. On the other hand, from Table 6 , it can be seen from this table that the values of $f^{\prime \prime}(0)$ and $\theta(0)$ are higher for $\operatorname{Pr}$ $=0.7$ than those for $\operatorname{Pr}=1$ and 7 .

Tables 7 and 8 show the values of $C_{f}$ and $\theta_{w}(x)$ for $\operatorname{Pr}=0.7, K=1$ (micropolar fluid) and various values of $\lambda$, respectively. It can be seen from these tables that as the parameter $\lambda$ increases, $C_{f}$ increases while $\theta_{w}(x)$ decreases. The actual value of $\lambda=\lambda_{k}$, which first gives no separation is difficult to determine exactly as it has to be found from the equations. However, the numerical solution indicates that the value of $\lambda_{k}$ which first gives no separation lies between 0.05 and 1 for fixed $\operatorname{Pr}=0.7$ and $K=1$.

Tables 9 and 10 present the values of the local skin friction coefficient $C_{f}$ and the wall temperature distribution $\theta_{w}(x)$ for $\lambda=1, K=1$ and various values of $\operatorname{Pr}$, respectively. It is found that, for fixed $\lambda=1$ and $K=1$, as Pr increases, both the $C_{f}$ and $\theta_{w}(x)$ decreases. On the other hand, for fixed $\mathrm{Pr}$, as $x$ increases, that is, from the lower stagnation point of the sphere, $x \approx 0$, and proceeds round the sphere up to the point, $x=120^{\circ}$, both the values of $C_{f}$ and $\theta_{w}(x)$ increase.

The velocity and temperature profiles near the lower stagnation point of the sphere, $x \approx 0$, for various values of $K$ with fixed $\operatorname{Pr}=7$ and $\lambda=1$ are plotted in Figures 2 and 3, respectively. From these figures, it is shown that as $K$ increases, the velocity profiles decrease and the temperature profiles increase. Figures 4 and 5 illustrate the velocity and temperature profiles near the lower stagnation point of the sphere, $x \approx 0$, for various values of $\lambda$ with fixed $\operatorname{Pr}=1$ and $K=$ 1 , respectively. We found that the velocity profiles increase while the temperature profiles decrease when the mixed 
TABLe 7: Values of the local skin friction coefficient $C_{f}$ for $\operatorname{Pr}=0.7$, $K=1$ and various values of $\lambda(\mathrm{NH})$.

\begin{tabular}{lcccccc}
\hline$x$ & \multicolumn{5}{c}{$\lambda$} \\
& 0.005 & 0.02 & 0.05 & 1 & 5 & 7 \\
\hline $0^{0}$ & 0.0000 & 0.0000 & 0.0000 & 0.0000 & 0.0000 & 0.0000 \\
$10^{0}$ & 2.2261 & 2.2280 & 2.2319 & 2.2995 & 2.5190 & 2.5862 \\
$20^{0}$ & 4.6769 & 4.6803 & 4.6870 & 4.8001 & 5.1915 & 5.3111 \\
$30^{0}$ & 7.0750 & 7.0797 & 7.0889 & 7.2419 & 7.7920 & 7.9602 \\
$40^{0}$ & 9.4615 & 9.4673 & 9.4789 & 9.6676 & 10.3657 & 10.5797 \\
$50^{0}$ & 11.6907 & 11.6976 & 11.7113 & 11.9338 & 12.7718 & 13.1966 \\
$60^{\circ}$ & 13.7553 & 13.7631 & 13.7788 & 14.0312 & 15.0807 & 15.8120 \\
$70^{0}$ & 15.7534 & 15.7622 & 15.7796 & 16.0600 & 17.4505 & 18.2886 \\
$80^{\circ}$ & 17.4679 & 17.4775 & 17.4965 & 17.8096 & 19.4869 & 20.3704 \\
$90^{\circ}$ & 19.0260 & 19.0362 & 19.0566 & 19.4335 & 21.2685 & 22.1885 \\
$100^{\circ}$ & & 20.2155 & 20.2370 & 20.6934 & 22.5758 & 23.5257 \\
$110^{\circ}$ & & & 21.1823 & 21.6963 & 23.5557 & 24.5200 \\
$120^{\circ}$ & & & & 22.1532 & 23.9418 & 24.8979 \\
\hline
\end{tabular}

TABLE 8: Values of the wall temperature distribution $\theta_{w}(x)$ for $\operatorname{Pr}=$ $0.7, K=1$ and various values of $\lambda(\mathrm{NH})$.

\begin{tabular}{lcccccc}
\hline$x$ & \multicolumn{5}{c}{$\lambda$} \\
& 0.005 & 0.02 & 0.05 & 1 & 5 & 7 \\
\hline $0^{0}$ & 3972.1601 & 997.6703 & 402.7344 & 24.9385 & 7.7892 & 6.3404 \\
$10^{0}$ & 14946.0423 & 3741.4930 & 1500.5887 & 78.2596 & 18.0482 & 13.3545 \\
$20^{0}$ & 15962.9645 & 3995.3036 & 1601.7763 & 82.9188 & 18.8185 & 13.8678 \\
$30^{0}$ & 16578.9876 & 4149.1161 & 1663.1461 & 85.8007 & 19.3253 & 14.2146 \\
$40^{\circ}$ & 17114.5642 & 4282.9142 & 1716.5875 & 88.3705 & 19.8096 & 14.5542 \\
$50^{0}$ & 17663.7427 & 4420.1691 & 1771.4575 & 91.0592 & 20.3424 & 14.8667 \\
$60^{0}$ & 18250.9445 & 4566.9709 & 1830.1793 & 93.9733 & 20.9019 & 15.0610 \\
$70^{0}$ & 18940.4194 & 4739.3755 & 1899.1698 & 97.4257 & 21.4484 & 15.3222 \\
$80^{\circ}$ & 19744.1335 & 4940.3723 & 1979.6231 & 101.4657 & 22.0515 & 15.7276 \\
$90^{0}$ & 20692.2273 & 5177.4964 & 2074.5533 & 106.1772 & 22.8095 & 16.2955 \\
$100^{\circ}$ & 5460.6204 & 2187.9111 & 111.7045 & 23.7877 & 17.0402 \\
$110^{0}$ & & & 2324.9537 & 118.3370 & 25.0444 & 17.9891 \\
$120^{\circ}$ & & & & 126.5161 & 26.6599 & 19.1931 \\
\hline
\end{tabular}

convection parameter $\lambda$ increases. Also, it is noticed that there are overshoots of the velocity profiles when $\lambda>1$ where these overshoots take place higher for $\lambda=10$ than for $\lambda=1$.

Figures 6 and 7 illustrate the velocity and temperature profiles near the lower stagnation point of the sphere, $x \approx 0$, for various values of $\operatorname{Pr}$ with fixed $K=2$ and $\lambda=1$, respectively. It is seen that, as the Prandtl number $\operatorname{Pr}$ increases, both the velocity and temperature profiles decrease. At large $\operatorname{Pr}(\gg 1)$, the thermal boundary layer is thinner than at smaller Pr. This is because for small values of $\operatorname{Pr}(\ll 1)$, the fluid is highly conductive. Physically, if $\mathrm{Pr}$ increases, the thermal diffusivity decreases and this phenomena leads to the decreasing of energy ability that
TABle 9: Values of the local skin friction coefficient $C_{f}$ for $\lambda=1$, $K=1$ and various values of $\operatorname{Pr}(\mathrm{NH})$.

\begin{tabular}{lccc}
\hline$x$ & & $\operatorname{Pr}$ & \\
\hline $0^{0}$ & 0.7 & 1 & 7 \\
$10^{0}$ & 0.0000 & 0.0000 & 0.0000 \\
$20^{\circ}$ & 2.2995 & 1.5053 & 0.1490 \\
$30^{\circ}$ & 4.8001 & 3.1609 & 0.3806 \\
$40^{\circ}$ & 7.2419 & 4.7598 & 0.5211 \\
$50^{\circ}$ & 9.6679 & 6.3723 & 0.7668 \\
$60^{\circ}$ & 11.9338 & 7.8549 & 0.8887 \\
$70^{\circ}$ & 14.0312 & 9.2817 & 1.0657 \\
$80^{0}$ & 16.0600 & 10.7771 & 1.3207 \\
$90^{\circ}$ & 17.8096 & 12.0495 & 1.4135 \\
$100^{\circ}$ & 19.4335 & 13.2069 & 1.6429 \\
$110^{\circ}$ & 20.6934 & 14.0239 & 1.6813 \\
$120^{\circ}$ & 21.6963 & 14.6790 & 1.8646 \\
\hline
\end{tabular}

TABLE 10: Values of the wall temperature distribution $\theta_{w}(x)$ for $\lambda=$ $1, K=1$ and various values of $\operatorname{Pr}(\mathrm{NH})$.

\begin{tabular}{lccc}
\hline$x$ & \multicolumn{3}{c}{$\operatorname{Pr}$} \\
\hline $0^{0}$ & 0.7 & 1 & 7 \\
$10^{\circ}$ & 78.9385 & 13.7810 & 1.2988 \\
$20^{\circ}$ & 82.9188 & 47.5798 & 3.4913 \\
$30^{\circ}$ & 85.8007 & 50.5583 & 3.8423 \\
$40^{\circ}$ & 88.3705 & 52.3905 & 4.0603 \\
$50^{\circ}$ & 91.0592 & 54.0104 & 4.2166 \\
$60^{\circ}$ & 93.9733 & 55.6998 & 4.3814 \\
$70^{\circ}$ & 97.4257 & 57.4598 & 4.5348 \\
$80^{\circ}$ & 101.4657 & 59.2523 & 4.6891 \\
$90^{\circ}$ & 106.1772 & 61.1798 & 4.8683 \\
$100^{\circ}$ & 111.7045 & 63.4915 & 5.0758 \\
$110^{\circ}$ & 118.3370 & 66.3902 & 5.3383 \\
$120^{\circ}$ & 126.5161 & 70.0617 & 5.6635 \\
\hline
\end{tabular}

reduces the thermal boundary layer. It is also noticed that there are overshoots of the velocity profiles when $\operatorname{Pr} \leq 1$ where these overshoots are higher for $\operatorname{Pr}=0.7$ than for $\operatorname{Pr}=1$.

\section{Conclusions}

In this paper, we have numerically studied the problem of mixed convection boundary layer flow from a solid sphere in a micropolar fluid, generated by Newtonian heating. It is shown that the mixed convection or buoyancy parameter $\lambda$, the material or micropolar parameter $K$, and the Prandtl number Pr affect the flow and heat transfer characteristics. We can conclude that (for the case of $\mathrm{NH}$ ): 


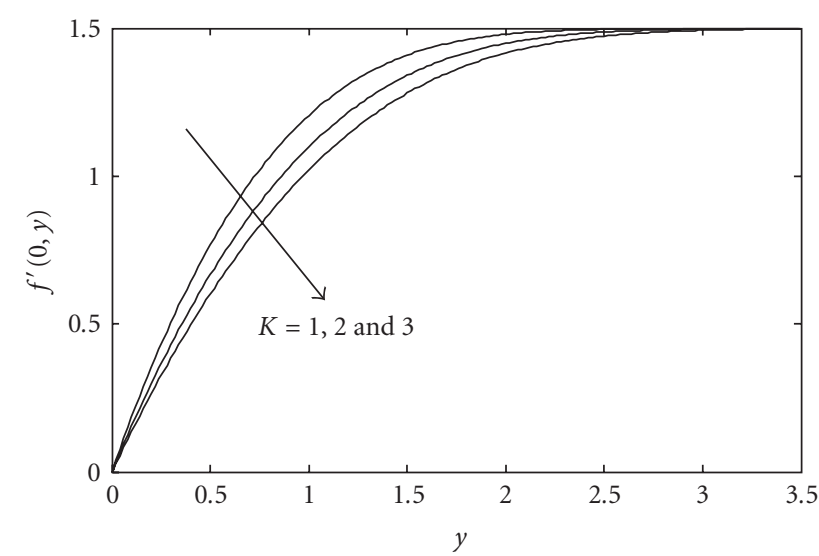

Figure 2: Velocity profiles for various values of $K$ with fixed $\operatorname{Pr}=$ 7 and $\lambda=1(\mathrm{NH})$.

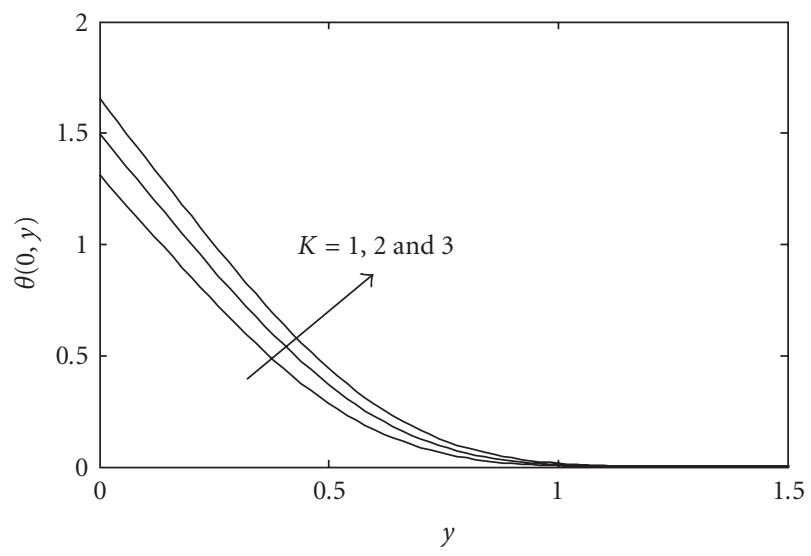

Figure 3: Temperature profiles for various values of $K$ with fixed $\mathrm{Pr}=7$ and $\lambda=1(\mathrm{NH})$.

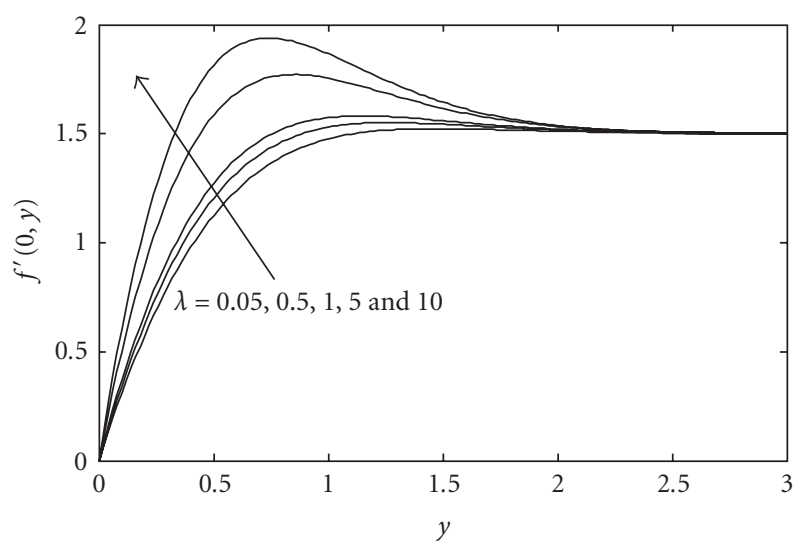

FIgURE 4: Velocity profiles for various values of $\lambda$ with fixed $\operatorname{Pr}=$ 1 and $K=1(\mathrm{NH})$.

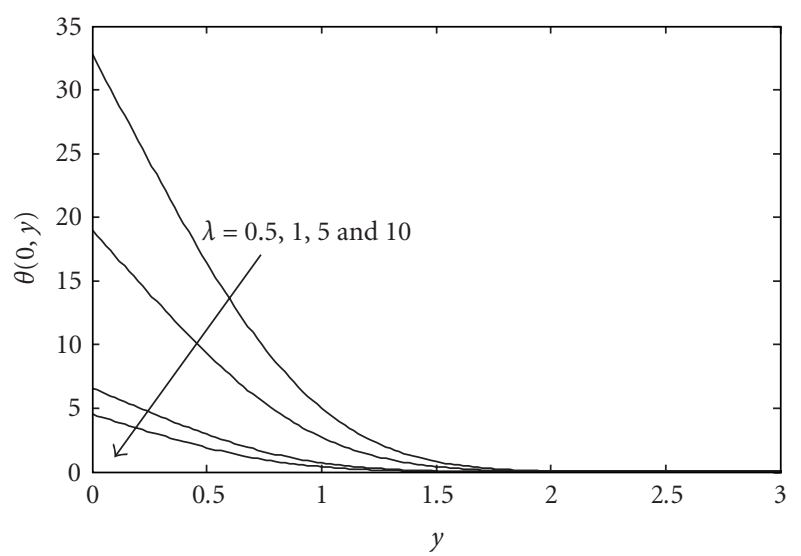

Figure 5: Temperature profiles for various values of $\lambda$ with fixed $\operatorname{Pr}=1$ and $K=1(\mathrm{NH})$.

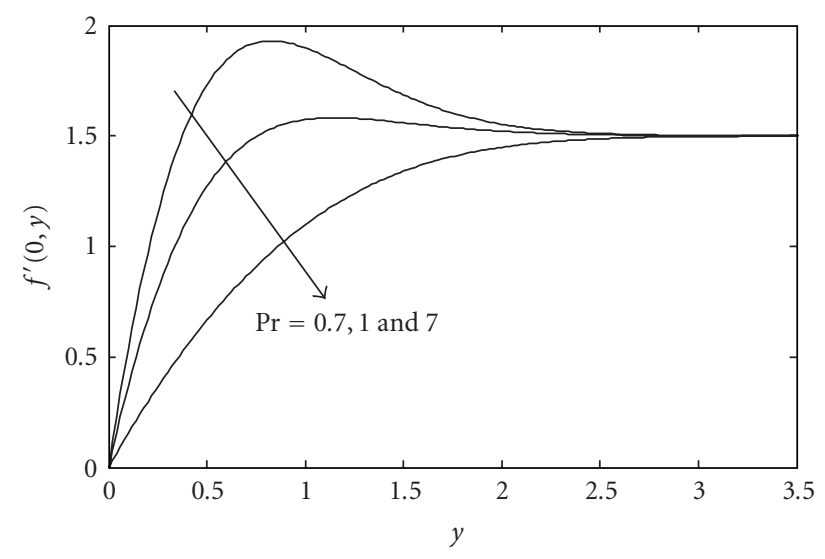

FIGURE 6: Velocity profiles for various values of Pr with fixed $K=$ 2 and $\lambda=1(\mathrm{NH})$.

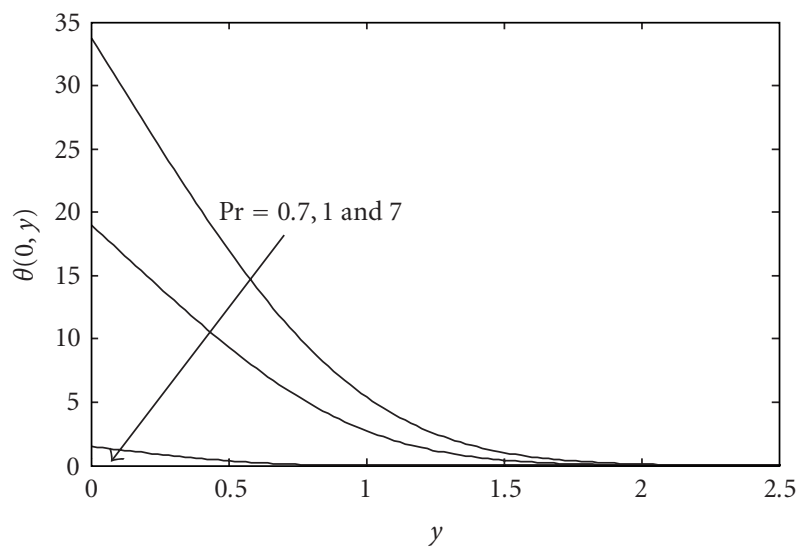

FIGURE 7: Temperature profiles for various values of Pr with fixed $K=2$ and $\lambda=1(\mathrm{NH})$. 
(i) when $\operatorname{Pr}$ and $K$ are fixed, an increase in parameter $\lambda$ leads to the decrease of both the heat transfer coefficient $-\theta^{\prime}(0)$ and the wall temperature $\theta(0)$;

(ii) when $\operatorname{Pr}$ and $\lambda$ are fixed, an increase in parameter $K$ leads to the decrease of the skin friction coefficient $f^{\prime \prime}(0)$ and the increase of the wall temperature $\theta(0)$;

(iii) when $\operatorname{Pr}$ and $K$ are fixed, an increase in parameter $\lambda$ leads to the increase of the skin friction coefficient $f^{\prime \prime}(0)$ and the decrease of the wall temperature $\theta(0)$;

(iv) when $K$ and $\lambda$ are fixed, an increase in parameter $\operatorname{Pr}$ leads to the decrease of the skin friction coefficient $f^{\prime \prime}(0)$ and the wall temperature $\theta(0)$;

(v) near the lower stagnation point of the sphere, the velocity profiles decrease while the temperature profiles increase when the parameter $K$ increases;

(vi) near the lower stagnation point of the sphere, the velocity profiles increase while the temperature profiles decrease when the parameter $\lambda$ increases;

(vii) near the lower stagnation point of the sphere, both the velocity and temperature profiles decrease when the Prandtl number Pr increases.

\section{Acknowledgment}

The authors gratefully acknowledge the financial supports received from the Ministry of Higher Education, Malaysia (UKM-ST-07-FRGS0036-2009) and a research grant (RDU 090308) from the Universiti Malaysia Pahang.

\section{References}

[1] A. C. Eringen, "Theory of micropolar fluids," Journal of Mathematics \& Mechanics, vol. 16, pp. 1-18, 1966.

[2] T. Ariman, M. A. Turk, and N. D. Sylvester, "Applications of microcontinuum fluid mechanics," International Journal of Engineering Science, vol. 12, no. 4, pp. 273-293, 1974.

[3] D. A. S. Rees and A. P. Bassom, "The Blasius boundarylayer flow of a micropolar fluid," International Journal of Engineering Science, vol. 34, no. 1, pp. 113-124, 1996.

[4] I. Pop, H. S. Takhar, and M. Kumari, "Free convection about a vertical wavy surface with prescribed surface heat flux in a micropolar fluid," Technische Mechanik, vol. 18, pp. 229-237, 1998.

[5] R. Nazar, N. Amin, and I. Pop, "Free convection boundary layer on an isothermal horizontal circular cylinder in a micropolar fluid," in Proceedings of the 12th International Heat Transfer Conference, vol. 2, pp. 525-530, Elsevier, Paris, France, August 2002.

[6] R. Nazar, N. Amin, and I. Pop, "Mixed convection boundary layer flow from a sphere with constant surface heat flux in a micropolar fluid," Journal of Energy, Heat and Mass Transfer, vol. 29, no. 8, pp. 1129-1138, 2002.

[7] R. Nazar, N. Amin, and I. Pop, "On the mixed convection boundary-layer flow about a solid sphere with constant surface temperature," The Arabian Journal for Science and Engineering, vol. 27, no. 2C, pp. 117-135, 2002.
[8] R. Nazar, N. Amin, and I. Pop, "Mixed convection boundary layer flow about an isothermal sphere in a micropolar fluid," International Journal of Thermal Sciences, vol. 42, no. 3, pp. 283-293, 2003.

[9] J. H. Merkin and I. Pop, "Conjugate free convection on a vertical surface," International Journal of Heat and Mass Transfer, vol. 39, no. 7, pp. 1527-1534, 1996.

[10] J. H. Merkin, "Natural-convection boundary-layer flow on a vertical surface with Newtonian heating," International Journal of Heat and Fluid Flow, vol. 15, no. 5, pp. 392-398, 1994.

[11] D. Lesnic, D. B. Ingham, and I. Pop, "Free convection boundary-layer flow along a vertical surface in a porous medium with Newtonian heating," International Journal of Heat and Mass Transfer, vol. 42, no. 14, pp. 2621-2627, 1999.

[12] D. Lesnic, D. B. Ingham, and I. Pop, "Free convection from a horizontal surface in a porous medium with Newtonian heating," Journal of Porous Media, vol. 3, no. 3, pp. 227-235, 2000.

[13] D. Lesnic, D. B. Ingham, I. Pop, and C. Storr, "Free convection boundary-layer flow above a nearly horizontal surface in a porous medium with Newtonian heating," Heat and Mass Transfer, vol. 40, no. 9, pp. 665-672, 2004.

[14] I. Pop, D. Lesnic, and D. B. Ingham, "Asymptotic solutions for the free convection boundary-layer flow along a vertical surface in a porous medium with Newtonian heating," Hybrid Methods in Engineering, vol. 2, pp. 31-40, 2000.

[15] R. C. Chaudhary and P. Jain, "Unsteady free convection boundary-layer flow past an impulsively started vertical surface with Newtonian heating," Romanian Journal of Physics, vol. 9, pp. 911-925, 2006.

[16] R. C. Chaudhary and P. Jain, "An exact solution to the unsteady free-convection boundary-layer flow past an impulsively started vertical surface with Newtonian heating," Journal of Engineering Physics and Thermophysics, vol. 80, no. 5, pp. 954-960, 2007.

[17] M. Z. Salleh, R. Nazar, and K. Ibrahim, "Mixed convection boundary layer flow near the lower stagnation point of a solid sphere with Newtonian heating," in Proceedings of the 7th WSEAS International Conference on System Science and Simulation in Engineering (ICOSSE '08), pp. 291-298, Venice, Italy, November 2008.

[18] M. Z. Salleh, R. Nazar, and I. Pop, "Forced convection boundary layer flow at a forward stagnation point with Newtonian heating," Chemical Engineering Communications, vol. 196, no. 9, pp. 987-996, 2009.

[19] T. Y. Na, Computational Methods in Engineering Boundary Value Problem, Academic Press, New York, NY, USA, 1979.

[20] T. Cebeci and P. Bradshaw, Physical and Computational Aspects of Convective Heat Transfer, Springer, New York, NY, USA, 1988.

[21] S. K. Jena and M. N. Mathur, "Similarity solutions for laminar free convection flow of a thermomicropolar fluid past a non-isothermal vertical flat plate," International Journal of Engineering Science, vol. 19, no. 11, pp. 1431-1439, 1981.

[22] G. S. Guram and A. C. Smith, "Stagnation flows of micropolar fluids with strong and weak interactions," Computers and Mathematics with Applications, vol. 6, no. 2, pp. 213-233, 1980.

[23] G. Ahmadi, "Self-similar solution of imcompressible micropolar boundary layer flow over a semi-infinite plate," International Journal of Engineering Science, vol. 14, no. 7, pp. 639646, 1976. 
[24] J. Peddieson Jr., "An application of the micropolar fluid model to the calculation of a turbulent shear flow," International Journal of Engineering Science, vol. 10, no. 1, pp. 23-32, 1972.

[25] M. Z. Salleh, S. Ahmad, and R. Nazar, "Numerical solutions of the forced convection boundary layer flow at a forward stagnation point," European Journal of Scientific Research, vol. 19, no. 4, pp. 644-653, 2008. 